\title{
Pyrolyse-flash de déchets ligno-cellulosiques en vue de leur valorisation par l'énergie solaire concentrée
}

\author{
$\left(^{*}\right)$ J. Lede, P. Berthelot, J. Villermaux, \\ (**) A. Rolin, H. François et X. Deglise \\ $\left(^{*}\right)$ Laboratoire des Sciences du Génie Chimique, C.N.R.S.-E.N.S.I.C., 1, rue Grandville, 54042 Nancy Cedex, France \\ $\left({ }^{* *}\right)$ Laboratoire de Photochimie Appliquée, E.R.A. n ${ }^{0} 136$ du C.N.R.S., Université de Nancy I, \\ C.O. 140, 54037 Nancy Cedex, France
}

(Reçu le 10 août 1979, révisé le 17 décembre 1979, accepté le 18 décembre 1979)

\begin{abstract}
Résumé. - On propose un procédé de valorisation de déchets de bois par pyrolyse flash grâce à l'énergie solaire concentrée. Une étude expérimentale est effectuée entre 700 et $1000^{\circ} \mathrm{C}$ avec de la sciure de pin Douglas au four électrique et au four à image dans des conditions voisines d'un concentrateur solaire. Il est possible, dans ces conditions, de produire avec des rendements atteignant $80 \%$, des mélanges gazeux riches en carbone monoxyde et hydrogène avec des proportions non négligeables d'hydrocarbures légers. On discute de l'intérêt du gaz sur les plans chimique et énergétique.

Abstract. - The purpose of this paper is to propose a method for the valorization of wood wastes by flash pyrolysis and use of concentrated solar energy. An experimental study has been made between 700 and $1000{ }^{\circ} \mathrm{C}$ with Douglas pine sawdust in electric and image furnace (similar conditions to a solar concentrator). In these conditions, we obtain $\mathrm{CO}$ and $\mathrm{H}_{2}$ rich gaseous mixtures with non negligible proportions of light hydrocarbons and with gasification yields up to $80 \%$. The energetical and chemical values of the gas are emphasized.
\end{abstract}

1. Introduction. - 1.1 LA BIOMASSE A DES FINS ÉNERGÉTIQUES. - L'approvisionnement des pays industriels en gaz naturel et produits pétroliers apparaît comme limité et les perspectives de découvertes de nouveaux gisements par des méthodes classiques sont incertaines [1]. Par l'unique procédé naturel de photosynthèse, l'énergie solaire est convertie en matériau végétal : la biomasse. Cette biomasse peut être transformée en gaz de substitution qui pourrait constituer une solution partielle à nos problèmes énergétiques, solution d'autant plus intéressante que la biomasse présente un caractère essentiellement renouvelable.

Deux grandes voies permettent de transformer la biomasse en gaz de substitution : la voie thermochimique et la voie biochimique.

Avec un taux de lignine important (30 à $40 \%$ ) et une structure particulière, le bois [2] ne semble pas devoir être valablement exploité par la voie biochimique, envisageable seulement pour des déchets d'exploitation agricole (fermentation de fumier, lisier, etc.). La voie thermochimique semble donc la mieux adaptée au bois, dont il est intéressant de chiffrer les ressources.

1.2 L'IMPORTANCE DES DÉCHeTS DE BOIS [3, 4]. Sur une superficie totale de 13480000 ha, les forêts françaises produisent 46,4 millions de $\mathrm{m}^{3}$ de bois (25,6 de feuillus et 20,8 de résineux).

La récolte totale de bois avoisinerait 31 à 32 millions de $\mathrm{m}^{3}$ dont 1 à 2 seraient autoconsommés (chauffage, etc.) et 29 consommés par les entreprises $\left({ }^{1}\right)$. Le volume global de déchets industriels est approximativement de 13 millions de $\mathrm{m}^{3}$.

Il serait donc tout à fait raisonnable, sans modifier ni diminuer les utilisations traditionnelles du bois, de disposer de 42 millions de $\mathrm{m}^{3}$, pour une valorisation énergétique, répartis de la façon suivante :

Déchets industriels $: 13$ millions de $\mathrm{m}^{3}$. Production non exploitée : 14 millions de $\mathrm{m}^{3}$. Production non recensée $\left({ }^{2}\right): 15$ millions de $\mathrm{m}^{3}$. (taillis, souches, etc.).

Compte tenu de la valeur énergétique du bois (en moyenne $4500 \mathrm{kcal} / \mathrm{kg}$ ), cette valorisation de 42 millions de $\mathrm{m}^{3}$ ou 29 millions de tonnes permettrait d'économiser $12 \mathrm{M}$ tep par an, soit $8 \%$ de notre consommation actuelle.

( ${ }^{1}$ ) Nous constatons qu'il n'est récolté en France que $69 \%$ de la production, à comparer avec des taux de récolte de $80 \%$ en R.F.A. et $90 \%$ dans les pays nordiques.

$\left({ }^{2}\right)$ Les chiffres précédemment cités concernent en moyenne des bois de diamètre supérieur à $7 \mathrm{~cm}$. 
1.3 LES TECHNIQUES DE VALORISATION ÉNERGÉTIQUE DU BOIS. - Il est possible d'envisager cinq techniques différentes de valorisation énergétique du bois : la combustion directe, la distillation, la liquéfaction, la gazéification, la pyrolyse.

- La combustion directe est mise en œuvre depuis toujours pour la production d'eau chaude, de vapeur et d'électricité. Son utilisation n'est envisageable que localement (aux alentours d'une zone forestière).

- La distillation $[5,6]$ permet la production de charbon de bois, de méthanol et d'acide acétique. Si cette technologie est très rentable à l'heure actuelle à cause de la demande en charbon de bois, elle ne peut être envisagée comme procédé industriel de valorisation énergétique. De plus, le rendement thermodynamique est médiocre.

- La liquéfaction du bois doit permettre à partir de déchets de faible granulométrie, d'aboutir à un pétrole synthétique en réduisant le matériau végétal sous pression (10 à $20 \mathrm{MPa})$ d'hydrogène, d'oxyde de carbone ou d'un mélange des deux $[7,8]$. Ce procédé qui fonctionne au stade pilote ne semble pas être rentable au point de vue énergétique. Il faut plutôt le considérer comme une source de produits chimiques élaborés (aromatiques par exemple).

- La gazéification du bois a été mise en œuvre à l'échelle industrielle pendant les deux dernières guerres mondiales et depuis cette époque [9], peu de progrès notables ont été faits si ce n'est en utilisant de l'oxygène à la place de l'air (réacteur Purox [10]). La plupart des gazogènes ainsi développés $[10,11]$ sont utilisés pour la valorisation énergétique des déchets urbains solides qui ne représentent en France que $10 \%$ de la matière végétale sèche issue du bois. Ces gazogènes fonctionnent tous selon le même principe. Ils comprennent une zone de pyrolyse et une zone de gazéification. La chaleur nécessaire à la gazéification du charbon de bois obtenu dans la zone de pyrolyse est fournie par des gaz de combustion à une température de $1000^{\circ} \mathrm{C}$. Ces gaz de combustion sont produits dans une chambre attenante au gazéificateur où sont brûlés les hydrocarbures obtenus au cours de la pyrolyse, le comburant étant l'oxygène.

L'inconvénient de ces procédés est de produire des gaz énergétiquement pauvres, du fait de la présence de dioxyde de carbone et parfois d'azote provenant de l'air.

- La pyrolyse : Il résulte de travaux effectués au cours des dernières années que l'on peut obtenir un gaz beaucoup plus riche en effectuant un chauffage extérieur du réacteur [12].

Certains auteurs ont montré d'autre part $[13,14,15]$ que la pyrolyse menée entre 1000 et $1700^{\circ} \mathrm{C}$ et avec des temps d'échauffement très courts $\left(0,2\right.$ à $\left.10^{-4} \mathrm{~s}\right)$ produisait de grandes quantités de gaz combustibles et peu de goudrons.

Il apparaît que les méthodes de pyrolyse, en présence ou non d'eau, méritent une étude plus appro- fondie en vue de leur éventuelle mise en œuvre optimale comme procédé de valorisation énergétique de la biomasse et en particulier du bois. La richesse du mélange gazeux escompté pourrait laisser envisager son utilisation soit comme produit primaire de substitution pour l'industrie chimique, soit comme combustible à haute valeur énergétique facilement transportable.

1.4 L'UTILISATION DE L'ÉNERGIE SOLAIRE. - La mise en œuvre de ce type de réaction nécessite un apport de chaleur extérieur et, comme l'ont montré certaines expériences, la quantité de résidus (goudrons et charbons) se trouve minimisée à condition d'effectuer un chauffage brutal du bois. Ces conditions suggèrent l'utilisation de l'énergie solaire concentrée permettant, d'une part, d'apporter gratuitement les calories nécessaires à la réaction et, d'autre part, de réaliser le choc thermique. Il est intéressant de noter que la combustion des produits de pyrolyse permettrait la restitution de l'énergie solaire stockée par photosynthèse lors de la formation du bois et de l'énergie stockée sous forme d'enthalpie de la réaction (endothermique) lors de la pyrolyse.

Remarquons enfin que jusqu'à présent tous les schémas d'utilisation de l'énergie solaire [16] séparaient la branche capteurs physiques de la branche biomasse. Le traitement thermochimique de la biomasse à l'aide de l'énergie solaire concentrée permettrait un couplage intéressant de ces deux grandes voies.

1.5 LeS OBJeCtifs DE CE PRÉSENT TRAVAIL. - Le manque de données expérimentales publiées nous a conduit, dans un premier temps, à effectuer une étude de l'influence d'un certain nombre de paramètres (température, granulométrie, taux d'humidité) sur la composition des gaz produits par la pyrolyse flash de sciure de pin Douglas en réacteur fermé, les calories étant apportées par un simple four électrique.

Parallèlement, d'autres expériences ont été menées en discontinu et en continu dans un réacteur placé dans des conditions comparables à celles régnant au foyer d'un concentrateur solaire (four à image)

Les résultats sont alors confrontés et la transposition de ces expériences au four solaire est discutée.

2. Aspect expérimental. - 2.1 TeCHNIQUeS EXPÉRIMENTALES. - 2.1.1 Produits utilisés. Techniques d'analyse. - La sciure de pin Douglas, choisie en raison de ses potentialités futures, a été fournie par le Centre National de la Recherche Forestière. La granulométrie correspond à des ouvertures de mailles de tamis variant de 0,14 à $0,5 \mathrm{~mm}$. Les échantillons sont séchés à $102^{\circ} \mathrm{C}$ durant $12 \mathrm{~h}$. Ils peuvent être utilisés tels quels ou après imprégnation de quantités connues d'eau pour étudier l'influence du taux d'humidité sur la réaction. Celui-ci est défini par le rapport entre la masse d'eau ajoutée et la masse de bois sec initial. Les analyses ont été effectuées par chromatographie 
à ionisation de flamme, thermistance et catharomètre, ainsi que par spectrophotométrie infrarouge.

2.1.2 Expériences effectuées au four électrique. Le montage schématisé sur la figure 1 permet d'effectuer la pyrolyse flash dans un réacteur en quartz placé dans un four électrique vertical. Les expériences ont lieu sous vide ou sous pression réduite de gaz inerte. Une quantité connue de bois est introduite dans une coupelle placée au-dessus du four, dans la partie froide du réacteur. Après avoir réalisé le vide dans l'installation, on retourne la coupelle et le bois tombe dans le réacteur chaud. On suppose que les grains de bois sont portés à une température proche de celle des parois. Un manomètre à mercure permet de suivre l'évolution de la pression. Lorsque celle-ci est stabilisée, le réacteur est mis en communication avec la rampe d'échantillonnage. Les gaz dont les coefficients de diffusion sont différents sont homogénéisés en les faisant circuler pendant $4 \mathrm{~min}$, par l'intermédiaire d'une pompe d'agitation en verre à travers trois ampoules d'analyse chromatographique et la cellule infrarouge à gaz. Les trois ampoules et la cellule sont ensuite fermées et branchées sur les appareils correspondant pour l'analyse. Ces conditions expérimentales seront publiées en détail dans un prochain article [17].

2.1.3 Appareillage simulant une installation solaire. Choix du réacteur.

- Le four à image [18].

Le montage [19] comporte une source lumineuse constituée par le cratère positif d'un arc électrique $(3 \mathrm{kVA})$ muni d'un système de rattrapage de l'usure des charbons. La source est placée au premier foyer d'un miroir elliptique. Le faisceau lumineux réfléchi est repris par un second miroir dont le second foyer est confondu avec le second foyer du miroir primaire. L'image de la source est ainsi formée au premier foyer du second miroir avec un grandissement égal à un. La puissance thermique récupérée est voisine de $150 \mathrm{~W}$. La section droite de la surface focale est proche de $0,2 \mathrm{~cm}^{2}$, ce qui correspond à une densité

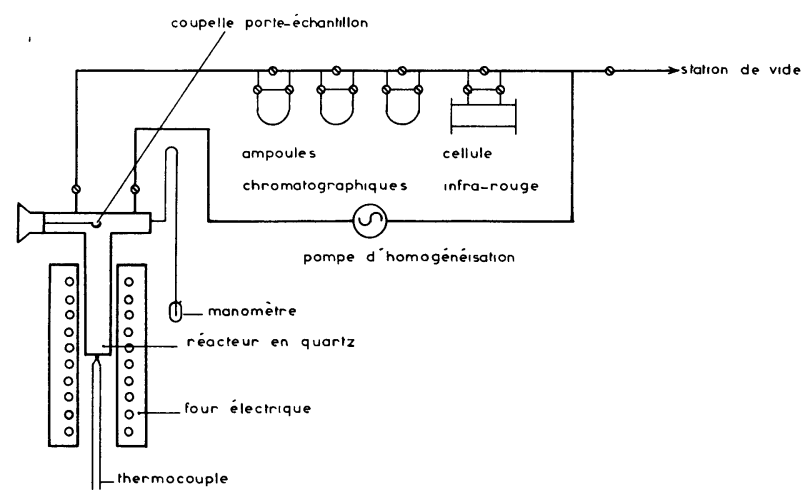

Fig. 1. - Schéma de principe de l'appareillage permettant la pyrolyse flash en discontinu au four électrique.

[Schematic diagram of apparatus for discontinuous flash pyrolysis in an electric furnace.] de flux de l'ordre de $800 \mathrm{~W} / \mathrm{cm}^{2}$, en supposant une répartition énergétique uniforme dans la tache. Cette valeur ainsi que celle de la puissance incidente sur le second miroir (de l'ordre de $1,5 \mathrm{~kW} / \mathrm{m}^{2}$ ) montrent que ce système permet de s'approcher des conditions de fonctionnement d'un concentrateur solaire, d'autant plus que la répartition énergétique du rayonnement reçu est proche de celle correspondant au rayonnement solaire [20]. Signalons qu'il a été possible dans ces conditions de réaliser la fusion de la zircone.

\section{- Le réacteur.}

Il convient de rechercher les conditions permettant de porter le plus rapidement possible une particule de bois à des températures proches de $1000^{\circ} \mathrm{C}$. Il s'agit d'un exemple typique de problème posé par la mise en œuvre d'un réacteur chimique solaire [21].

Chaque particule de bois peut être chauffée :

soit par transfert de chaleur au contact d'un fluide (vapeur d'eau) ou d'une surface solide située dans une cavité absorbante,

soit par absorption directe du rayonnement solaire lors du passage de la particule dans la zone focale, par chute libre ou guidée, par transport pneumatique ou en lit fluide.

La bonne émissivité du bois $\left(0,93\right.$ à $38^{\circ} \mathrm{C}$ [22]) alliée à ses médiocres qualités conductrices nous ont conduit à utiliser, dans le présent travail, la seconde technique.

Les expériences ont été faites en discontinu et en continu. Dans tous les cas, la connaissance expérimentale de la température atteinte par la sciure est d'un accès très difficile.

- Montage expérimental en discontinu (Fig. 2).

Une petite cavité en alumine, d'un volume intérieur proche de $0,2 \mathrm{~cm}^{3}$ est placée au foyer image du miroir secondaire. Cette cavité est située à l'intérieur d'un ballon en verre pyrex de $320 \mathrm{~cm}^{3}$ où règne le vide ou une pression contrôlée d'argon. Un échantillon de bois (sec ou mouillé) de masse connue est placé

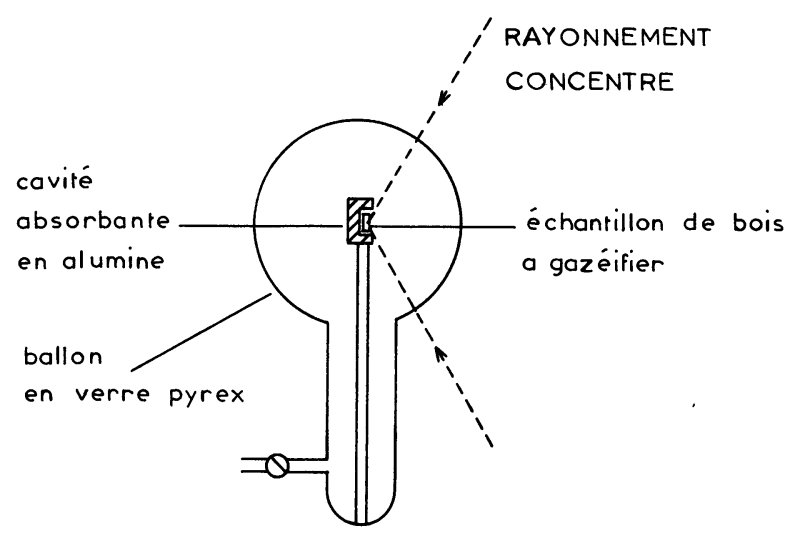

Fig. 2. - Schéma de principe du réacteur permettant la pyrolyse flash en discontinu au four à image.

[Schematic diagram of reactor for discontinuous flash pyrolysis in an image furnace.] 
dans la cavité. Il est soumis au rayonnement lumineux pendant le court temps nécessaire à la réaction (inférieur à $1 \mathrm{~s}$ ). Après réaction, le ballon est mis en communication avec le système d'analyse.

Les échantillons sont constitués soit de grains de sciure retenus sur des tamis de 0,14 à $2 \mathrm{~mm}$, soit de copeaux de $60 \mu \mathrm{m}$ d'épaisseur et de masses comprises entre 0,5 et $1,2 \mathrm{mg}$.

- Montage expérimental en continu (Fig. 3).

La sciure traverse le volume focal en chute libre, guidée par une surface d'alumine courbe placée derrière cette zone. La régularité de l'alimentation est assurée par un vibreur placé à la sortie de la réserve de sciure. Pour des diamètres de grains compris entre 0,14 et $0,4 \mathrm{~mm}$ et un débit de bois de l'ordre de $0,02 \mathrm{~g} / \mathrm{min}$, une expérience peut être poursuivie pendant une vingtaine de minutes, sans qu'on observe d'échauffement important du ballon. Lors des expériences sous vide, le gaz de réaction s'accumule dans tout l'appareil et on relève l'augmentation de pression. Lors des expériences sous pression atmosphérique, le gaz s'échappe par le manomètre. Les essais ont été effectués uniquement avec du bois sec, étant donné la difficulté de transporter de la sciure de bois mouillé.

Plus de détails peuvent être trouvés dans la référence [19].

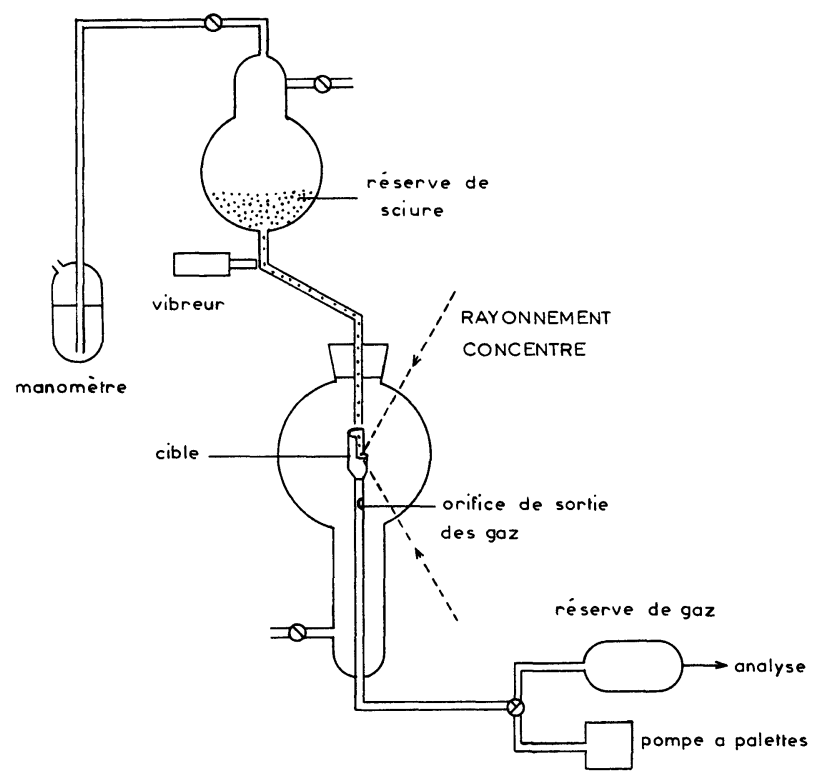

Fig. 3. - Schéma de principe de l'appareillage permettant la pyrolyse flash en continu au four à image.

[Schematic diagram of apparatus for continuous flash pyrolysis in an image furnace.]

3. Résultats expérimentaux. - Désignons par $X$ le taux de gazéification défini ainsi :

$$
X=\frac{\text { Masse des produits gazeux formés }}{\text { Masse de l'échantillon initial }} \text {. }
$$

La figure 4 montre que $X$ augmente avec la température et le taux d'humidité. Dans nos conditions expérimentales (expériences faites au four électrique), $X$ varie de 50 à $78 \%$.

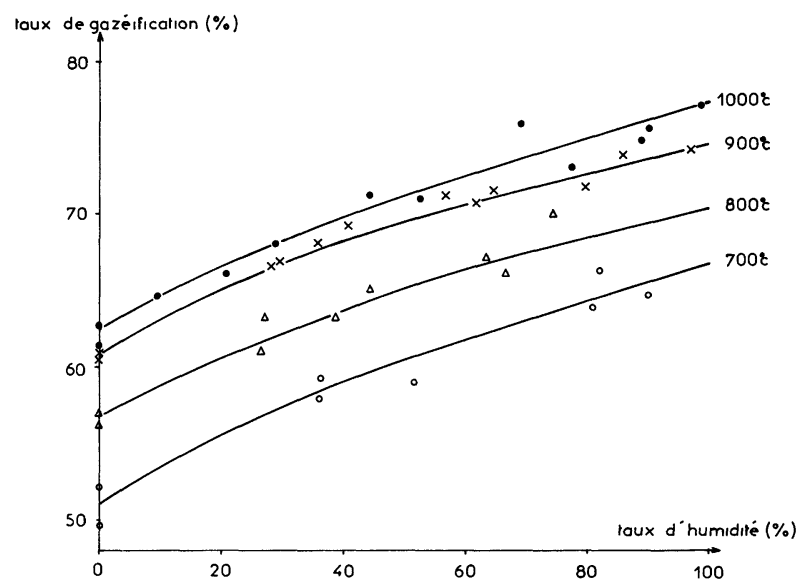

Fig. 4. - Variations expérimentales du taux de gazéification $X$ en fonction du taux d'humidité $(\%)$ pour différentes températures.

[Experimental variations of gasification yield $X$ with centesimal moisture content for different temperatures.]

La figure 5 et le tableau I indiquent les compositions du mélange gazeux obtenu à différentes températures et taux d'humidité au four électrique et au four à image.
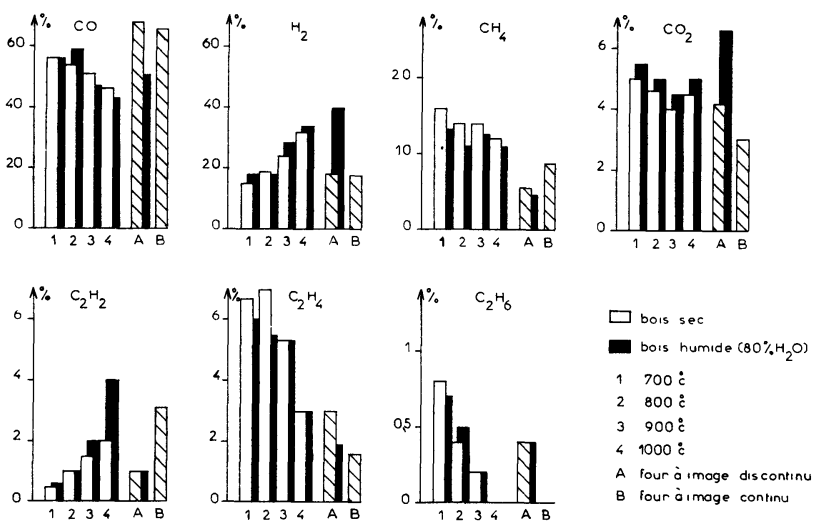

Fig. 5. - Composition centésimale du mélange gazeux obtenu expérimentalement par pyrolyse flash de bois sec et mouillé à différentes températures au four électrique et au four à image.

[Centesimal composition of gases evolved by flash pyrolysis of dry and wet wood at different temperatures in electric and image furnace.]

Nous avons représenté sur la figure 6 le volume des gaz dégagés par gramme de bois (conditions TPN) en fonction de la température. Le volume gazeux obtenu est plus grand avec du bois humide, ce qui corrobore les observations faites sur le taux de gazéification. On constate également que pour des températures légèrement supérieures à $1000^{\circ} \mathrm{C}$, on récu- 
Tableau I.

\begin{tabular}{|c|c|c|c|c|c|c|c|c|c|c|c|}
\hline \multirow[b]{2}{*}{$\%$ en vol. } & \multicolumn{8}{|c|}{ Four électrique } & \multicolumn{2}{|c|}{ Discontinu } & \multirow{2}{*}{$\begin{array}{l}\text { Continu } \\
0 \% \text { eau }\end{array}$} \\
\hline & $\begin{array}{l}700{ }^{\circ} \mathrm{C} \\
0 \% \text { eau }\end{array}$ & $\begin{array}{c}700{ }^{\circ} \mathrm{C} \\
80 \% \text { eau }\end{array}$ & $\begin{array}{l}800{ }^{\circ} \mathrm{C} \\
0 \% \text { eau }\end{array}$ & $\begin{array}{c}800{ }^{\circ} \mathrm{C} \\
79 \% \text { eau }\end{array}$ & $\begin{array}{l}900^{\circ} \mathrm{C} \\
0 \% \text { eau }\end{array}$ & $\begin{array}{c}900{ }^{\circ} \mathrm{C} \\
87 \% \text { eau }\end{array}$ & $\begin{array}{l}1000^{\circ} \mathrm{C} \\
0 \% \text { eau }\end{array}$ & $\begin{array}{l}1000{ }^{\circ} \mathrm{C} \\
90 \% \text { eau }\end{array}$ & $90 \%$ eau & $0 \%$ eau & \\
\hline $\mathrm{CO}$ & 56 & 56 & 54 & 59 & 51 & 47 & 46,5 & 43 & 45,7 & 67,9 & 65,7 \\
\hline $\mathrm{CO}_{2}$ & 5 & 5,5 & 4,6 & 5 & 4 & 4,5 & 4,5 & 5 & 6,6 & 4,2 & 3 \\
\hline $\mathrm{H}_{2}$ & 15 & 18 & 19 & 18 & 24 & 28,5 & 32 & 34 & 39,8 & 18 & 17,8 \\
\hline $\mathrm{CH}_{4}$ & 16 & 13,2 & 14 & 11 & 14 & 12,5 & 12 & 11 & 4,6 & 5,5 & 8,8 \\
\hline $\mathrm{C}_{2} \mathrm{H}_{4}$ & 6,7 & 6 & 7 & 5,5 & 5,3 & 5,3 & 3 & 3 & 1,9 & 3 & 1,6 \\
\hline $\mathrm{C}_{2} \mathrm{H}_{6}$ & 0,8 & 0,7 & 0,4 & 0,5 & 0,2 & 0,2 & 0 & 0 & 0,4 & 0,4 & 0 \\
\hline $\mathrm{C}_{2} \mathrm{H}_{2}$ & 0,5 & 0,6 & 1 & 1 & 1,5 & 2 & 2 & 4 & 1 & 1 & 3,1 \\
\hline $\begin{array}{l}\text { Taux de gazéification } \\
\text { en } \%\end{array}$ & 52 & 66 & 60 & 70 & 62,5 & 74 & 62 & 77 & 60 & à & $75 \%$ \\
\hline
\end{tabular}

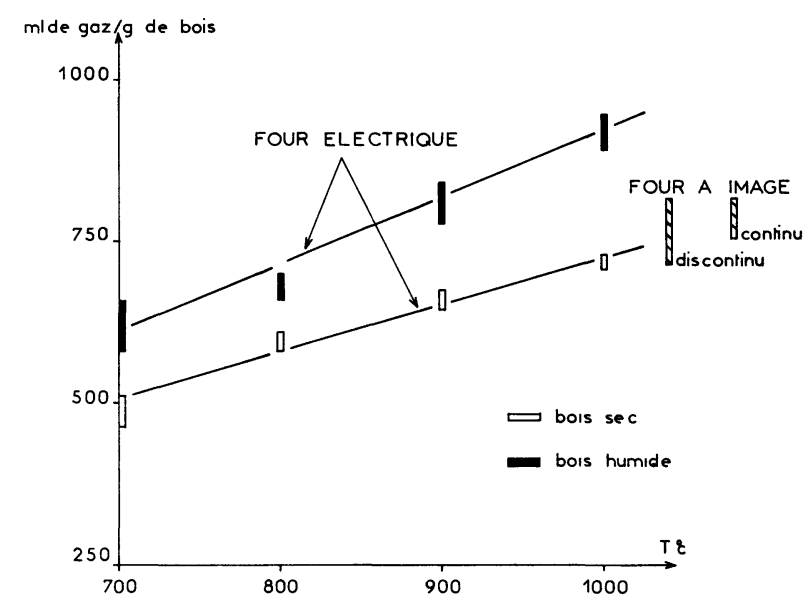

Fig. 6. - Variations expérimentales du volume de gaz dégagé par pyrolyse flash de $1 \mathrm{~g}$ de bois en fonction de la température.

[Experimental variations of the volume of gases evolved by flash pyrolysis of $1 \mathrm{~g}$ of wood at different temperatures.]

père approximativement $11 \mathrm{de}$ gaz par gramme de bois. Il est à noter que cet ordre de grandeur correspond à des conditions expérimentales sans récupération des goudrons et charbons. Les expériences effectuées au four à image donnent des valeurs comparables et laissent supposer une température proche de $1000^{\circ} \mathrm{C}$ dans ces conditions.

4. Discussion. - 4.1 Rôle de L'EAU. - Sur le plan de la composition chimique des gaz, l'eau ne semble pas avoir de rôle important dans les expériences au four électrique. Au four à image, il semblerait que la présence d'eau augmente le pourcentage d'hydrogène alors que celui du monoxyde de carbone diminue.

La figure 4 montre par contre que la présence d'eau a une influence indéniable sur le taux de gazéification.
Des analyses en spectrométrie de masse sur des gaz formés à partir d'échantillons de bois humidifiés avec de l'eau lourde ont montré des traces de deutérium moléculaire, les hydrocarbures, quant à eux, n'étant pas deutérés.

Ces résultats montrent que l'eau a un rôle chimique dans la réaction, mais également une part probablement importante sur le plan physique.

\section{2 CONFRONTATION DES RÉSUltATS. TEMPÉRATURE.} - Le tableau I montre un parallélisme certain entre les résultats obtenus au four électrique et au four à image, les températures atteintes par les particules dans ces dernières conditions devant avoisiner $1000^{\circ} \mathrm{C}$. Cet ordre de grandeur est confirmé par les expériences de Goheen et coll. [14], ces auteurs ayant opéré entre 1040 et $1760^{\circ} \mathrm{C}$.

Il est intéressant de noter que nos résultats sont tout à fait en accord avec ceux obtenus récemment par M. J. Antal au four solaire de $1 \mathrm{MW}$ d'Odeillo, dans des conditions proches des nôtres [23].

Ces constatations montrent que les résultats obtenus au four électrique ou au four à image peuvent être valablement utilisés pour concevoir une installation solaire. Des difficultés expérimentales ont été rencontrées avec le four à image étant donné la petitesse de la tache focale et la difficulté de canaliser les particules de bois dans cette zone. Si l'on utilise un concentrateur classique, il serait donc intéressant de concevoir un système d'étalement et d'homogénéisation de la zone focale (au détriment de la valeur de la densité de flux et donc de la vitesse de chauffage).

L'utilisation de centrales à tour est également envisageable.

On peut essayer d'évaluer l'ordre de grandeur du choc thermique imposé aux particules, donc la vitesse de chauffage dans le système à four à image. Pour une particule de $0,14 \mathrm{~mm}$ de diamètre supposée 
sphérique et soumise à une densité de flux de $800 \mathrm{~W} / \mathrm{cm}^{2}$, on peut calculer une vitesse de chauffage initiale de l'ordre de $4 \times 10^{5}{ }^{\circ} \mathrm{C} \mathrm{s}^{-1}$.

4.3 INFLUENCE DE LA GRANULOMÉTRIE. - Les expériences au four électrique et en continu au four à image ont été effectuées avec des particules retenues sur des tamis à mailles variant de 0,14 à $0,5 \mathrm{~mm}$. Les expériences en discontinu ont porté sur des échantillons de masse comprise entre 0,6 et $16 \mathrm{mg}$. Aucune influence sensible n'a pu être observée, ce qui est confirmé par les travaux de Maa [24].

Faisons l'approximation d'une cinétique globale d'ordre 1 [25]. Avec une énergie d'activation de $30 \mathrm{kcal} / \mathrm{mole}$ et un facteur de fréquence de $7 \times 10^{9} \mathrm{~s}^{-1}$ [26], on peut calculer à $1000^{\circ} \mathrm{C}$ un temps caractéristique de réaction de l'ordre de $2 \times 10^{-5} \mathrm{~s}$. Ce temps est notablement inférieur au temps caractéristique de transfert de chaleur dans un grain (de l'ordre de $r^{2} / \alpha$ où $r$ est le rayon d'un grain et $\alpha$ le coefficient de diffusivite thermique) proche de $10^{-2} \mathrm{~s}$ pour notre granulométrie. La vitesse de pyrolyse est donc largement limitée par le transfert interne de chaleur. On pourrait ainsi imaginer un mécanisme d'attaque par pelage progressif du grain, les parties extérieures étant seules chauffées.

L'état de division des échantillons utilisés (essentiellement sous forme de sciure) se justifie à l'échelle du laboratoire et pour des études plus fondamentales de granulométrie. Lors de la conception d'installations plus importantes, il conviendra de ne pas oublier de tenir compte du coût nécessaire au broyage des déchets disponibles.

4.4. INTÉRÊT DU GAZ OBTENU SUR LE PLAN CHIMIQUE. - Deux possibilités de valorisation des gaz peuvent être envisagées sur le plan chimique :

- Le gaz est particulièrement riche en $\mathrm{CO}$ et $\mathrm{H}_{2}$ et pourrait donc constituer un point de départ de préparation de gaz de synthèse.

- On constate également la présence non négligeable d'hydrocarbures légers $\left(\mathrm{C}_{2} \mathrm{H}_{4}\right.$ et $\left.\mathrm{C}_{2} \mathrm{H}_{2}\right)$. La proportion d'éthylène est plus importante vers 700 $800^{\circ} \mathrm{C}$ dans des conditions où le taux de gazéification $X$ est malheureusement assez faible. La recherche de catalyseurs pourrait s'avérer fructueuse. Par contre, les proportions d'acétylène augmentent avec la température en même temps que $X$. Il serait donc intéressant de miser sur la préparation de ce composé, d'autant plus que, comme l'ont montré Goheen et coll. [14], des rendements en acétylène proches de $40 \%$ en masse par rapport au bois pourraient être théoriquement escomptés.

4.5 INTÉRÊT DU GAZ OBTENU SUR LE PLAN ÉNERGÉTIQUE. - A partir des compositions centésimales du gaz obtenu (Tableau I), et des chaleurs de combustion de chacun des constituants, on peut calculer une chaleur de combustion du mélange gazeux rapporté à $1 \mathrm{~m}^{3}$ de gaz (conditions TPN). La figure 7 montre

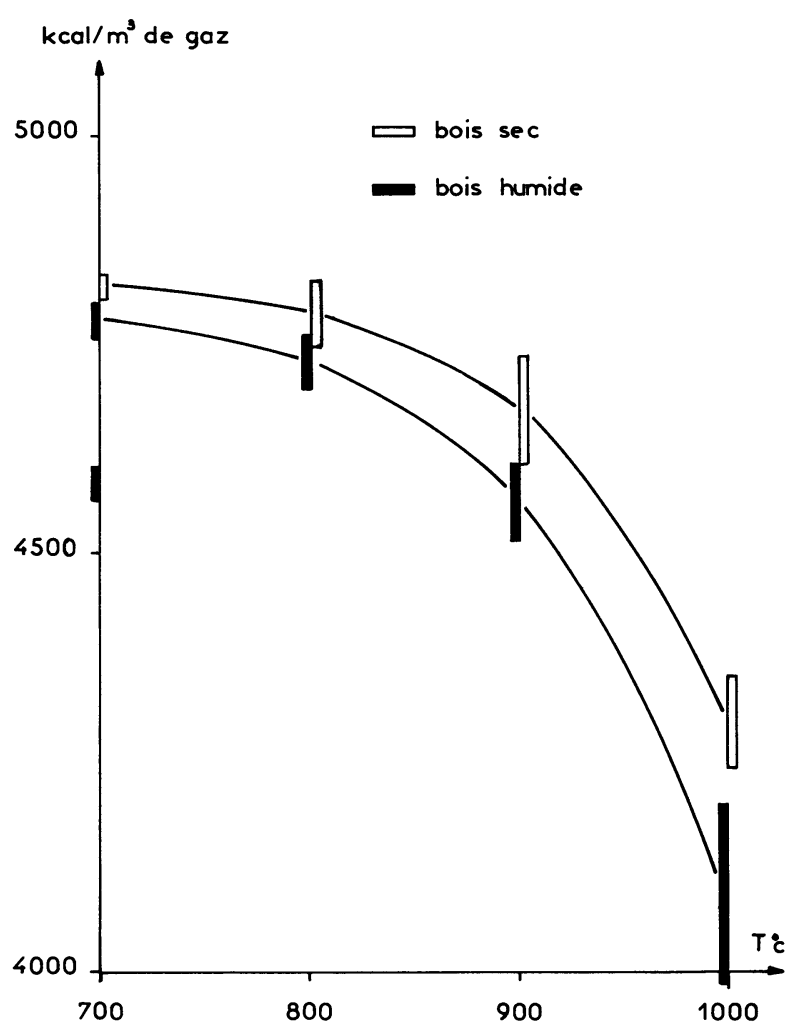

Fig. 7. - Chaleur libérée par la combustion de $1 \mathrm{~m}^{3}$ du mélange gazeux obtenu par pyrolyse flash du bois à différentes températures (volumes ramenés en conditions TPN).

[Energy produced by the combustion of $1 \mathrm{~m}^{3}$ of gaseous mixture evolved by flash pyrolysis of wood at different temperatures (TPN conditions).]

que ces valeurs diminuent lorsque la température augmente. Ce résultat s'explique par le fait que lorsque la température augmente, les proportions relatives de méthane, d'éthane et d'éthylène (à haut pouvoir calorifique) diminuent. Cette constatation est en accord avec les résultats prévus par d'autres auteurs [27]. Les valeurs obtenues sont cependant largement supérieures à celles correspondant à l'utilisation de gazogènes de type courant $\left(1000\right.$ à $2000 \mathrm{kcal} / \mathrm{m}^{3}$ en moyenne) [28].

Il est intéressant (Fig. 8) d'exprimer la chaleur de combustion des gaz en la rapportant à l'unité de masse de bois initial.

On constate que les valeurs obtenues sont toujours inférieures à celles correspondant à la combustion de $1 \mathrm{~kg}$ de bois de pin Douglas $(4500 \mathrm{kcal} / \mathrm{kg}$ : valeur déterminée au laboratoire en bombe calorimétrique). Sur le plan purement énergétique, aucun stockage de chaleur n'a donc été réalisé dans la phase gazeuse. Par contre, il n'est pas interdit de prévoir que la limite des $4500 \mathrm{kcal}$ puisse être dépassée en améliorant le taux de gazéification. Un tel résultat peut être atteint :

- Soit en augmentant encore la température de pyrolyse du bois, ce qui impose un choc thermique 


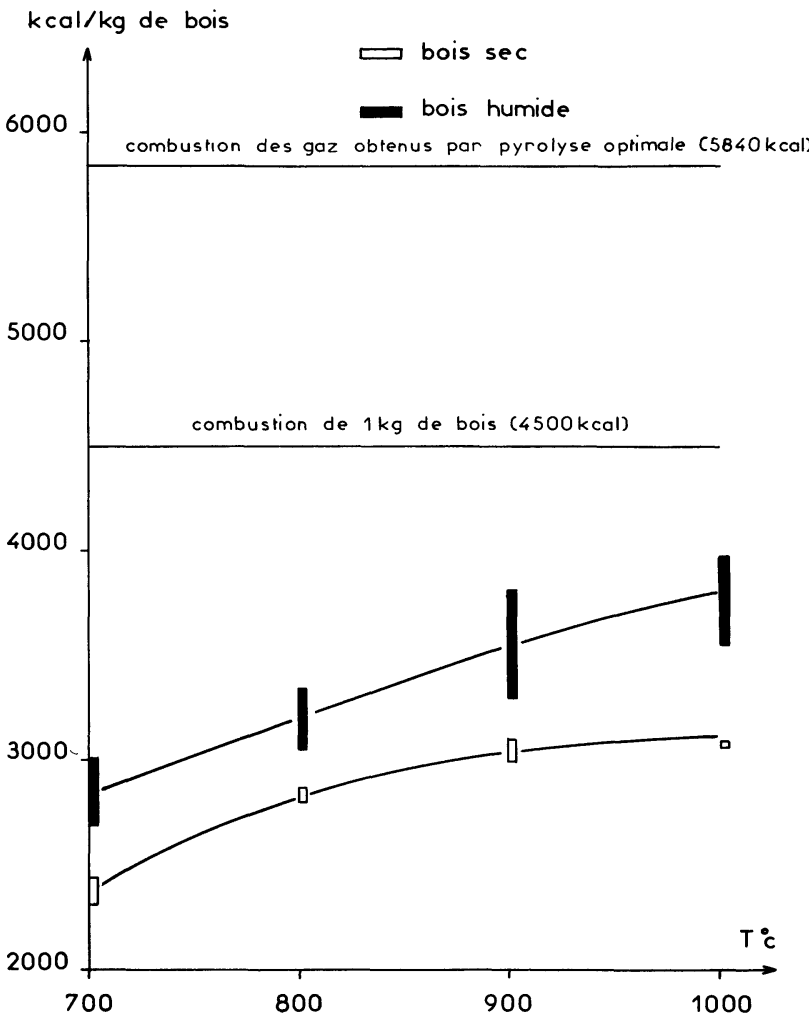

Fig. 8. - Chaleur libérée par la combustion du mélange gazeux obtenu par pyrolyse flash de $1 \mathrm{~kg}$ de bois à différentes températures.

[Energy produced by the combustion of gaseous mixture evolved by flash pyrolysis of $1 \mathrm{~kg}$ of wood.]

très important afin de minimiser les réactions aux températures intermédiaires.

- Soit par l'utilisation de catalyseurs, ceux-ci pouvant jouer également sur la sélectivité de la réaction.

- Soit en conservant des domaines de températures comparables à ceux explorés ici, mais en mettant en œuvre la réaction dans un réacteur continu permettant, outre le choc thermique initial, un séjour prolongé des produits dans une zone chaude soit un recyclage des goudrons en vue de leur gazéification propre [29].

Il est intéressant d'estimer une limite supérieure de l'énergie ainsi récupérable en supposant une réaction totale $(X=100 \%)$. Il est difficile d'anticiper sur la composition du mélange gazeux à conversion totale en présence d'eau ; mais on pourrait imaginer qu'un gaz contenant uniquement $\mathrm{CO}$ et $\mathrm{H}_{2}$ fournirait une chaleur de combustion maximale. Compte tenu de la formule brute du bois $\mathrm{CH}_{1,44} \mathrm{O}_{0,66}$, on peut calculer une chaleur de combustion d'un tel mélange égale à

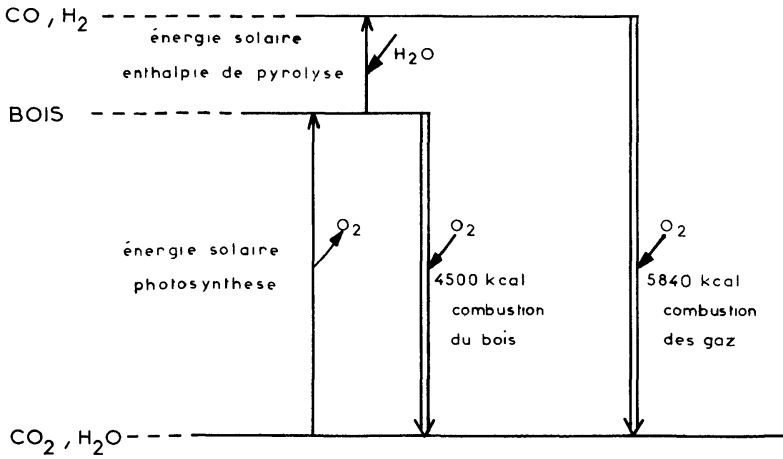

Fig. 9. - Représentation schématique de la quantité d'énergie solaire stockée pour $1 \mathrm{~kg}$ de bois par photosynthèse et par pyrolyse en présence d'eau.

[Schematic diagram representing the storage of solar energy during photosynthesis and pyrolysis of $1 \mathrm{~kg}$ of wood (wet).]

$5840 \mathrm{kcal} / \mathrm{kg}$ de bois. Dans ces conditions, l'enthalpie de la réaction de pyrolyse totale pourrait être estimée proche de $5840-4500=1340 \mathrm{kcal} / \mathrm{kg}$ de bois (limite supérieure).

Ainsi (Fig. 9), lors de la combustion du mélange gazeux obtenu dans ces conditions optimales, on peut penser récupérer l'énergie solaire stockée lors de la photosynthèse et celle fournie par le concentrateur solaire dans lequel a été effectuée la pyrolyse, soit au total $5840 \mathrm{kcal} / \mathrm{kg}$ de bois. Par rapport à la combustion de $1 \mathrm{~kg}$ de bois, il résulterait un gain d'énergie de $30 \%$.

Un rapide calcul montre qu'avec une enthalpie de réaction de $1340 \mathrm{kcal} / \mathrm{kg}$ de bois, il serait possible de traiter $640 \mathrm{~kg}$ de bois à l'heure dans une installation solaire de $1 \mathrm{MW}$. On pourrait espérer produire dans ces conditions $1200 \mathrm{~m}^{3}$ de gaz à l'heure. Il s'agit là d'estimations très optimistes qui supposent tous les rendements égaux à 1 .

En conclusion de ce paragraphe, on peut dire que si l'on désire un gaz à haute valeur énergétique, il est plus intéressant d'opérer au voisinage de 700 à $800^{\circ}$ (Fig. 7) au détriment du taux de gazéification et des problèmes posés par la présence d'importants résidus; par contre, la recherche de conditions plus sévères en température permettrait de s'approcher d'une gazéification totale du bois en fournissant un gaz énergétiquement plus pauvre, mais en réalisant un intéressant stockage d'énergie.

Remerciements. - Nous remercions le Centre National de la Recherche Forestière et la D.G.R.S.T. qui a accordé une aide au Laboratoire de Photochimie Appliquée, ainsi que MM. Richard, Tournier et Michelot pour leur aide sur le plan analytique et technique. 


\section{Bibliographie}

[1] Alich, J. A. et Imman, R. E., « Effective Utilization of Solar Energy to Produce Clean Fuel ». Final Report, NSF Grant $n^{\circ} 38723$ S.R.I. Project 2643, Stanford Research Institute (June 1974).

[2] Chartier, P. et DupuY, P., Compte rendu du Colloque de la Société Française de Microbiologie. Toulouse (1978).

[3] Brenac, L., Rev. For. Fr. 28 (1976) 30.

[4] Guillon, P., Rev. For. Fr. 28 (1976) 38

[5] BUGGE, G., Industrie der Holzdestillations produkte 6 (1927)

[6] Guillemonat, A., Le bois matière première de la chimie moderne (1942).

[7] Appell, H. R. et Miller, R. D., Conversion of Cellulosic Wastes to Oil. Bureau of Mines. Report of Investigation (1975).

[8] Boocock, D. G. B., Mackay, D., McPherson, M., Nadeau, S. et Thurier, R., Can. J. Chem. Eng. 57 (1979) 98.

[9] Delaunay, R., "Le Méthanol de Synthèse à partir du Bois, Réalisation Française ». Centre de Perfectionnement Technique. Fascicule no 1725 . Déc. 1946.

[10] Anderson, J. E., "Solid Refuse Dispasol Process and Apparatus ». U.S. Patent 3, 729, 298; April 24, 1973.

[11] Hammond, V. L., "Pyrolysis. Incineration Process for Solid Waste Disposal ». Battelle Pacific Northwest Laboratories, Richland, Washington; Dec. 1972.

[12] Prahacs, S., Barclay, H. G. et Bhatia, S. P., Pulp and Paper Magazine Can., 72 (1971) 199.

[13] Antal, M. J. Jr., U.S. Patent 3, 993, 458 ; Nov. 23, 1976.
[14] Goheen, D. W. et Henderson, J. I., Cellul. (hem. Technol. 12 (1978) 363.

[15] Antal, M. J., Bulletin of Atomic Scientists, 59 (1976).

[16] Groupe de Bellevue. Projet ALTER (1978).

[17] Rolin, A., François, H., Tournier, A., Richard, C. et DEGLISE, X., à paraître.

[18] Chaudron, G. et Trombe, F., Les Hautes Températures et leurs Utilisations en Physique et en Chimie. Tome I (Masson) 276 (1973).

[19] BeRTHELOT, P., Rapport interne. L.S.G.C.-E.N.S.I.C., Nancy (1979).

[20] Foex, M., Journal des Recherches du C.N.R.S. 65631 (1963).

[21] Villermaux, J., Entropie 85 (1979) 25.

[22] Sacadura, J. F., Initiation aux Transferts Thermiques. Technique et Documentation. Paris (1978).

[23] Antal, M. J., Communication personnelle.

[24] MAA, P., Ph. D. Dissertation. W. Virginia University, Morgontown W. A. (1971)

[25] Tran, D. Q. et RaI, C., Fuel 57 (1978) 293.

[26] Lewellen, P. C., Peters, W. A. et Howard, J. B., 6th Symposium on Combustion. Combustion Institute MIT, Cambridge. Mass (1976).

[27] Lewis, F. M., Solid Waste Processing Conference. Boston Mass (1976).

[28] Meunier, J., Gazéification et oxydation des Combustibles (Masson) 1978

[29] Lede, J., Verzaro, F. et Villermaux, J., à paraître. 